\title{
El capital relacional como recurso diferencial y valioso para las empresas
}

\section{Relational capital as a differential and valuable resource for companies}

María Yolanda Laverde Guzmán ', Carlos Alberto Almanza Junco ², Dustin Tahisin Gómez Rodríguez ${ }^{3}$, Claudia Liliana Serrano Junco ${ }^{4}$

INFORMACIÓN DEL

\section{ARTÍCULO}

Fecha de recepción: 6 de Marzo de 2020.

Fecha de aceptación: 4 de Junio de 2020

${ }^{1}$ MBA, Universidad de Viña del Mar. Docente-investigadora, Universitaria Agustiniana-Colombia.

E-mail:

maria.laverde@uniagustiniana.edu.co Código ORCID:

https://orcid.org/0000-0002-4395-0108

2 Doctorando en Administración, Universidad de Celaya. Docenteinvestigador, Universidad Militar Nueva Granada-Colombia.

E-mail: carlos.almanza@unimilitar.edu.co Código ORCID:

https://orcid.org/0000-0002-4561-4941

Doctorado en Agrociencias, Universidad de la Salle. Docenteinvestigador, Universitaria AgustinianaColombia.

E-mail:

Dustin.gomez@uniagustiniana.edu.co Código ORCID:

https://orcid.org/0000-0001-5359-2300

${ }^{4}$ Magíster en Docencia, Universidad de la Salle. Docente-investigadora, Corporación Universitaria Minuto de Dios-Colombia.

E-mail: claudia.serrano.j@uniminuto.edu Código ORCID:

https://orcid.org/0000-0001-6844-2735

CITACIÓN: Laverde Guzmán, M.Y., Almanza Junco,C.A., Gómez Rodríguez, D.T.,\& Serrano Junco, C.L.(2020). El capital relacional como recurso diferencial y valioso para las empresas. Podium, 37, 57-70.

\section{ENLACE DOI:}

http://dx.doi.org/10.31095/podium.202 0.37 .5

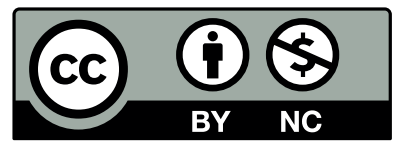

\section{Resumen}

Este artículo tiene por objeto analizar las posibles dinámicas entre el capital relacional y la red de valor de las pymes. La metodología es de corte cuantitativo y transversal, la cual inició con una extensa revisión de literatura sobre las variables de capital relacional en función de los aliados, proveedores, clientes, redes de valor, confianza, reputación, así como de los elementos que potencian o frenan sus desarrollos. Se concluye que hay relación entre el capital relacional y la red de valor, por lo tanto, el capital relacional es valorado como un recurso diferencial y valioso para aquellas empresas que lo potencian, lo cual es consistente cuando se asocia a la teoría de recursos y capacidades, en el sentido propio que los recursos intangibles, así como las capacidades de la empresa (para este caso específico el capital relacional) potencian la generación y obtención de resultados superiores.

\section{Palabras Clave:}

Capital relacional, redes de valor, gestión del conocimiento, pymes, proveedores, innovación.

Clasificación JEL: D80; D83; M50.

\section{Abstract}

This article aims to analyze the possible dynamics between relational capital and the value network of SMEs. The methodology is quantitative and cross-sectional, which began with an extensive literature review on the variables of relational capital based on allies, suppliers, customers, value networks, trust, reputation, as well as the elements that enhance or they hold back their developments. It is concluded that there is a relationship between relational capital and the value network, therefore, relational capital is valued as a differential and valuable resource for those companies that promote it, which is consistent when associated with the theory of resources and capabilities, in the sense that intangible resources, as well as the capabilities of the company (in this specific case, relational capital) enhance the generation and obtaining of superior results.

\section{Keywords:}

Relational capital, value networks, knowledge management, SMEs, suppliers, innovation.

JEL Classification: D80; D83; M50.

\section{7}

PODIUM No. 37, Junio 2020, pp. 57-70

(C) Universidad Espíritu Santo - UEES

ISSN: 1390-5473 e-ISSN: 2588-0969 


\section{Introducción}

Como resultados de los mercados altamente competitivos, las empresas se han visto obligadas a potencializar $\mathrm{y}$ rentabilizar al máximo sus activos; un activo particularmente efectivo para responder a este desafío es el conocimiento. Lo anterior supone que de la habilidad que tengan las empresas de gestionar el conocimiento para hacer frente a los retos que surgen a diario, dependerá la generación de la ventaja en la explotación de la información derivada del mercado (Losada, Bonilla, y Gómez, 2018). Por consiguiente, el conocimiento es y será en un recurso organizacional estratégico y la generación de ventajas competitivas estará alineada a la forma como este se gestione. Cuando este activo se gestiona intencionalmente, los miembros, así como la organización, aprenden de sus propias experiencias, creando mejores maneras de llevar a cabo procedimientos establecidos, lo que en últimas genera ciclos virtuosos que producen múltiples beneficios sociales, económicos y financieros (Gómez y Rincón,2016).

En consecuencia, el presente artículo, retoma esta visión sobre la gestión de conocimiento visto a través de su componente capital relacional y las ventajas que tiene su aplicación en la dinamización de las redes de valor. Para ello, se divide en una introducción en la que se desglosa las categorías utilizadas como sus líneas argumentativas. Prosigue con la metodología, continua con los resultados del ejercicio investigativo, la discusión y por último unas breves conclusiones.

\section{Revisión de literatura}

\section{Evolución del capital relacional}

Al revisar las tendencias administrativas y de gestión en las organizaciones en las últimas décadas, se puede identificar un gran avance en los procesos y manejos organizacionales, tendientes a ser cada vez más competitivos, para captar la atención de clientes (Brunet, 2009). Sin embargo, cuando se aborda este objetivo, se puede ver como los gustos y expectativas de los clientes ingresan también en esta dinámica relación, sin poder calibrar la próxima tendencia, que permita estar a tono con los mercados deseados. En este sentido, responder a las demandas que impone el siempre cambiante orden social, cultural y económico en el contexto global, sería la meta de toda organización, pero implica estar a la vanguardia del conocimiento como factor diferenciador (Rubio, Téllez, y Gómez, 2019; Dutton y Duncan, 1987). Por lo tanto, y tomando como derrotero la visión organizacional, el concepto conocimiento y capital relacional ha pasado de una visión típicamente mercantil en función de ser uno más de los recursos con que cuentan las organizaciones, a ser el recurso que dinamiza toda la organización tanto dentro como fuera de ella (Ciprés y Llusar, 2004; Lander, 2001; Teece, 1998; Spender, 1996). En consecuencia, el capital relacional ha sido definido como "la estructura externa de la organización, compuesta de la cartera de clientes, relaciones con los 
proveedores, bancos y accionistas, acuerdos de cooperación, alianzas estratégicas, tecnológicas, de producción y comerciales, las marcas comerciales y la imagen de la empresa" (Tejedo y Ferraz, 2016,p. 6). De igual modo, lo conforman la red de negocios que permiten establecer relaciones de valor, mayor avance y fortalecimiento en sus integrantes, transmisión de conocimientos, desarrollo de proveedores, economías de escala, llevando a las organizaciones a una espiral de crecimiento, haciéndolas más competitivas en la medida que más se articulen y relacionen.

\section{Capital intelectual}

Este concepto se ha capitalizando en las organizaciones a partir de la gestión del conocimiento, debido a su importancia a nivel competitivo, como un proceso de mejora continua que permite su desarrollo y aplicación, facilitando la solución de problemas de manera sostenible, colectiva y pertinente; dando lugar a la generación de documentación, usabilidad y transferencia en el flujo de formación, pero también a la creación de nuevas experiencias enmarcadas en valores, reforzando la identidad corporativa y convirtiendo saberes en un recurso estratégico, que da ventaja competitiva como factor diferenciador de la organización, que pasa de una economía de la información a una basada en el conocimiento, soportada desde luego por diversos capitales (humano, relacional y estructural) los cuales en sí mismo representan el activo intangible más importante de la organización (Aja, 2002). De allí que el capital intelectual representa los recursos no financieros que permiten ser más competitivos, dando respuestas a las necesidades de los diversos mercados, pasando del conocimiento personal al conocimiento organizacional, añadiendo valor a todos los stakeholders que conforman la red de servicios de las empresas (Rodríguez y Alfaro,2016). Por consiguiente, el capital intelectual juega a favor de las diversas instituciones a nivel micro y macro, en un contexto globalizado, que avanza rápidamente sin dar tregua a cometer errores, que en el largo plazo resultan en grandes pérdidas en los mercados meta de las organizaciones (Estrategia Magazine, 2018). De ahí que sea preciso, estar al ritmo constante que impone la dinámica económica, produciendo bienes o servicios que ofrezcan experiencia de valor cada vez más innovadoras, en el menor tiempo posible, rentables y competitivas, con calidad, servicio, precio, participación en el mercado, sin perder de vista la gente y una manera de lograr esto es a través de la integración de su red de valor.

\section{Redes de valor}

La cadena de valor o red de valor en los términos aceptados por el Consejo de Profesionales de Gestión de la Cadena de Suministro (CSCMP), como máximo ente rector de la logística en el mundo; lo concibe como el flujo que atraviesa de manera transversal los procesos de clase mundial de planeación, abastecimiento, producción, entrega y gestión de los retornos, mediante los redes de información y de materiales, cuya suma de esfuerzos es el resultado que percibe el 
cliente, siendo la calidad de los productos el reflejo de la calidad de las personas que participaron de manera directa o indirecta en dichos procesos (Estrategia Magazine, 2018).

\section{Integración de la red de valor}

Entendiendo la red de valor como una herramienta o medio sistemático, que permite analizar las fuentes que otorgan ventaja competitiva a una empresa, se puede dividir en actividades estratégicamente relevantes con el propósito de comprender el costo que genera su comportamiento e identificar las fuentes potenciales que hacen la diferencia en términos de competitividad, siendo la logística el proceso que permite su articulación mediante los flujos de inventarios y de información, fundamental en la organización para superar las expectativas de los clientes internos y externos (Kotler y Armstrong, 2012). Por ende, la logística en la cadena de suministro, hoy llamada cadena de valor, entendida como la combinación de procesos, funciones o actividades y relaciones de valor, o caminos por los cuales fluyen productos, servicios, transacciones financieras dentro y entre empresas, involucrando a todas las personas del mundo que la hacen posible (Gattorna, 2015). Sin embargo, su elemento clave son las personas que la impulsan y le dan vida, haciéndola un sistema viviente, a partir de sus comportamientos que la humanizan.

\section{Capital relacional como integrador de la red de valor}

La necesidad del capital relacional en las organizaciones está directamente relacionada con la capacitación y desarrollo de competencias del capital humano, influyendo de manera directa en los niveles de innovación y generación o transmisión de conocimientos, que favorezcan la integración. Precisamente, Mayorga, Ruíz, Mantilla y Mayolema (2015), establecen que las pymes se caracterizan por un bajo nivel de formación en los colaboradores, con escaso empoderamiento frente a procesos y procedimientos, desfavoreciendo la calidad, eficiencia y competitividad a nivel latinoamericano, asimismo Vargas, Villareal, López y Rodríguez (2014), revisan los factores que inciden en la baja competitividad de las organizaciones, enfatizando que estos resultados se asocian con la cultura de innovación, relevancia del capital humano y capital intelectual, llegando a determinar la gran necesidad que tienen todas las organizaciones de fomentar estos aspectos, a través de cambios en sus sistemas de gestión, basados en modelos cognitivos y procesos de aprendizaje, que reorienten la cultura, confianza y sentido de compromiso de los colaboradores, mediante un clima organizacional sano.

De igual modo, se puede identificar como la integración de colaboradores con su capital relacional, son relevantes en la organización, dejando al factor humano como fuente primaria de innovación y mejora continua, es así como se ha pasado de una mezcla de 50/50 infraestructura y sistemas de información, para tener una nueva redistribución 45/45/10, donde el primer porcentaje corresponde al recurso humano, seguido de la tecnología para dar una pequeña 
fracción a la infraestructura (Gattorna, 2009), siendo responsables de crear relaciones y alianzas de valor que den origen a nuevas habilidades, y permitan realinear de manera efectiva la red, generando nuevos modelos comerciales y por ende ventaja competitiva (Polo, Fernández, Bargsted, Ferguson y Rojas, 2017). En consecuencia, el capital relacional permite la integración de los empresarios, mitigando situaciones que minan la competitividad en las pymes (González y Gálvez, 2008). Ya que, al aplicar teorías de emprendimiento a las redes empresariales, logrando beneficios colaborativos, que favorecen el trabajo en equipo y la sinergia, el aprovechamiento de tecnologías, recursos, $\mathrm{y}$, por ende, la colaboración en la gestión de los diversos procesos que trae consigo iniciar un negocio. Por otra parte, al hablar de capital relacional, es preciso tener presente el papel que desempeñan las pymes en la economía de un país, pues aportan cerca del 95\% de empleos directos, impactando la economía en diversos aspectos macroeconómicos y a la sociedad. Por lo tanto, no es casual que varias instituciones públicas o privadas propongan diferentes programas con el fin de apoyar los procesos de investigación y desarrollo dirigidos a potencializar las pymes. Sin embargo, pareciera que el mensaje no llega y al final se desaprovechan todos estos esfuerzos, acorde con el trabajo de Vargas et al. (2014), donde muestra como esta situación se repite en varios países, llevando a este tipo de organizaciones al borde de la quiebra, de ahí que la integración de la red de valor, sea relevante para mejorar los procesos de investigación, desarrollo e innovación $(\mathrm{I}+\mathrm{D}+\mathrm{I})$ permitiendo mayores niveles de competitividad y fortalecimiento para los microempresarios.

\section{Descripción del modelo tradicional}

En la Figura 1 se puede identificar como desde el concepto tradicional se pretende dar solución al sistema, sin considerar las personas que son las conductoras de los flujos de información o de bienes y servicios que se gestiona desde la

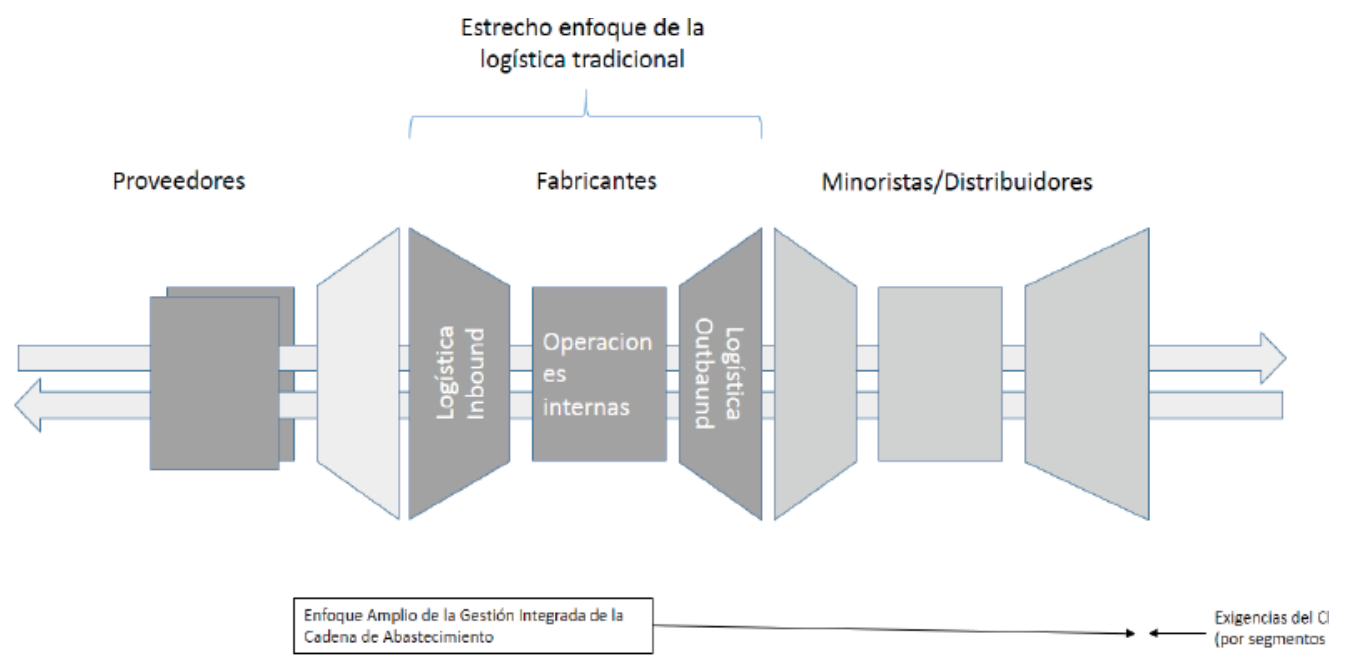

Figura 1. Puesta en práctica del concepto tradicional de la cadena de abastecimiento.

Fuente: Gattorna, 2015, p. 7. 


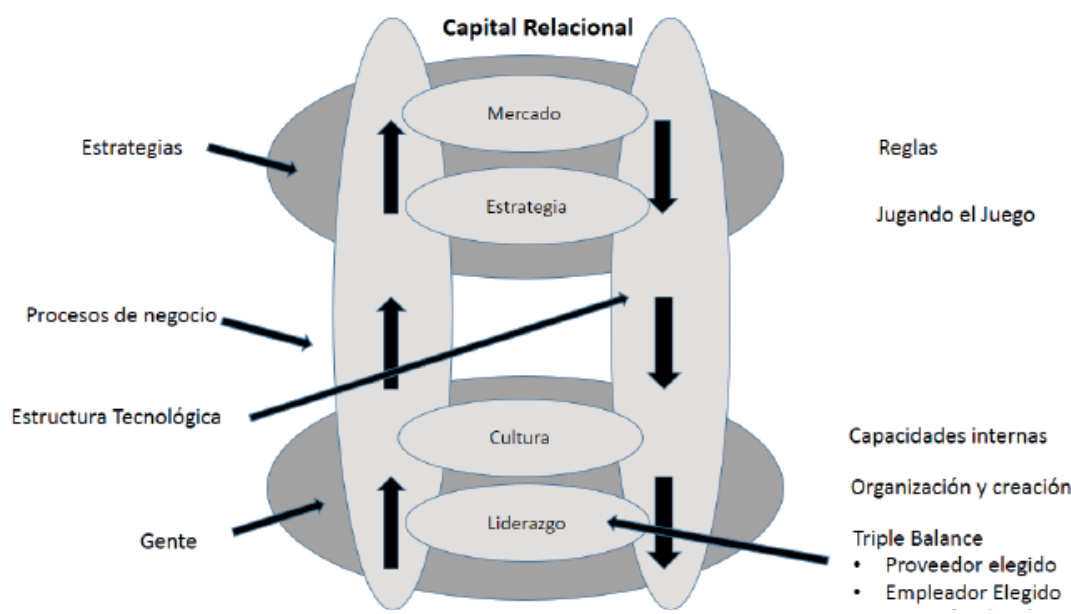

Figura 2. Alienación de la red de valor inmersa en el capital relacional. Fuente: Basado en el diagrama de alineación dinámica (Gattorna 2009, p.12).

logística en la red de valor, representados en los activos duros como son: Sistemas, infraestructura y equipos, sin reconocer los activos intangibles representados en las personas que imprimen ese sentido humano y relacional como factor diferenciador de la red, y por el cual los clientes están dispuestos a pagar (Gaitán, 2015; Pérez y Cortez 2009; Nonaka y Takeuchi, 1999).

Sin embargo, para que el equipo de colaboradores se comprometa de manera incondicional con las metas y niveles de servicio que las organizaciones persiguen, son necesarios estilos de liderazgo basados en fuertes conceptos culturales, fundamentados en principios y valores, constituyendo una cultura de las organizaciones que sirva de cohesionador de la red de valor, como se muestra en la Figura 2, lo cual implica estar en sintonía con los clientes tanto internos como externos, permitiendo logros colectivos como el triple balance que de manera individual sería muy difícil de lograr

Ciclo logístico a lo largo de la red de valor

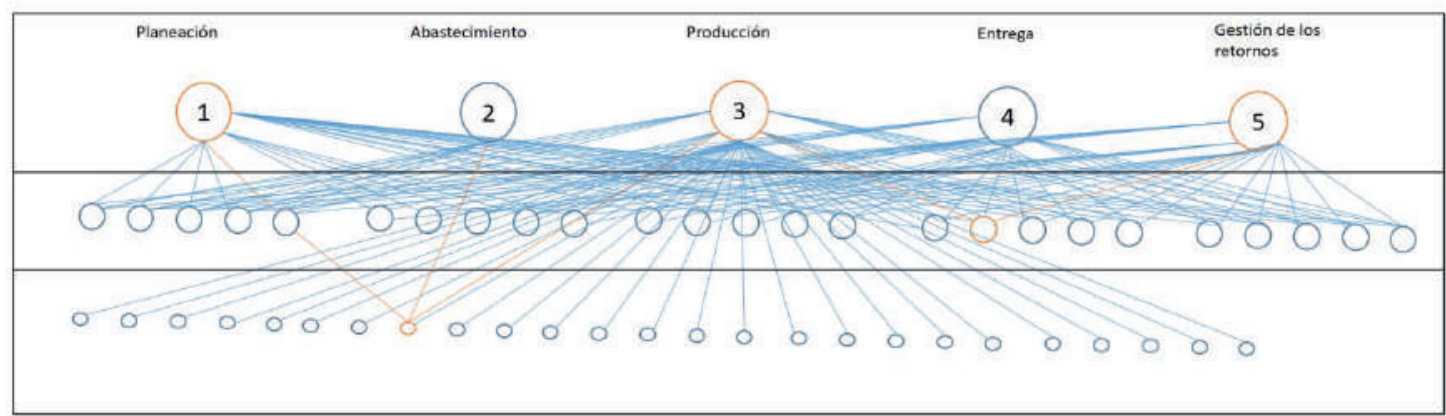

Figura 3. Ciclo logístico en la red de valor.

Fuente: Elaboración propia. 
según Contreras y Barbosa (2013) y Blanchard (2007).

Al revisar la Figura 3, se puede ver como la cadena de valor, en realidad es una red de valor donde los procesos de clase mundial se desarrollan de manera indistinta desde cualquier punto de la red, aguas arriba o aguas abajo, de manera interdependiente del nivel donde se encuentren el cliente que al final es el cliente de todos, teniendo en claro que estas conexiones son entre personas, que gracias a sus relaciones de valor, permiten que se den diversas transacciones de bienes y servicios, adicional a la generación de valor para toda la red (Figueroa, 2011).

También se puede ver en la Figura 3, que está representado hasta el tercer nivel, como al gestionar correctamente el capital relacional, se logran mayores niveles de competitividad con menores esfuerzos, poniendo de plano la integración, a través de desarrollo del proveedores, mediante la transmisión de conocimientos entre partners o aliados estratégicos, facilitando el manejo de inventarios acorde con las necesidades de la empresas, sobre todo las más pequeñas que no poseen el músculo financiero para comprar en volumen, pero que en alianza con otras, logran mejores precios al aprovechar economías de escala, lo cual redunda en beneficios para todas las partes involucradas (Capello y Faggian,2005). Adicional al manejo de inventarios también se encuentran casos en los cuales se pueden manejar las entregas, a través de los mismos proveedores, optimizando recorridos, maximizando el aprovechamiento efectivo de la flota de transportes, dando como resultado de estos esfuerzos, costos más competitivos para el cliente final, que ayudan a dinamizar la demanda, y, por ende, la rentabilidad de todos los stakeholders de la red de valor (Ahumada,2001).

\section{Metodología}

Este trabajo se desarrolló bajo los criterios de una investigación empírica, cuantitativa, y de corte transversal, la cual inició con una extensa revisión de literatura referente a las variables capital relacional en función de los aliados, los proveedores y los clientes, redes de valor relación, confianza, reputación, así como de los elementos que potencian o frenan sus desarrollos. La recolección de datos se hizo mediante cuestionarios auto administrados con una escala tipo Likert (1932) de 5 opciones de uno (1) a cinco (5), donde uno (1) indica totalmente en desacuerdo y cinco (5) indica totalmente de acuerdo; tres (3) es una valoración media, desde el mes de febrero a finales de octubre del 2018 en el territorio colombiano, particularmente dirigido a los niveles tácticos y estratégicos. Se obtuvieron en total 403 formularios, de los cuales de rechazaron por incompletitud 81, es decir más del $75 \%$ fueron válidos. El tratamiento de los datos se hizo con el apoyo del software estadístico Real Stats, siguiendo la recomendación de Anderson y Gerbing (1988) para abordar las propiedades psicométricas del modelo de medida a través de un Análisis Factorial Confirmatorio (AFC). 
La propuesta inicial del modelo sugería 50 ítems: Relaciones con clientes (6), relación con proveedores (6), relación con aliados (6), reputación (6), red social (4), visión compartida (6), confianza y apoyo social (4), y red de valor (12). Una validez de contendido por juez experto redujo el número de ítems a $35 \mathrm{y}$ con el AFC, algunos ítems con cargas bajas o residuales altos fueron eliminados, generando un modelo final de medición de 25 variables observables: Capital relacional referido a clientes (3), capital relacional referido a proveedores (3), relación con aliados (3), reputación (3), red social (2), visión compartida (3), confianza y apoyo social (2), y red de valor (6). De igual manera, se nutre con la metodología cualitativa desde el método de revisión documental (Gómez, Carranza y Ramos, 2017 ;2017º; 2016).

\section{Resultados}

La Tabla 1 presenta los resultados del AFC y los índices de bondad ajustados, los cuales muestran en general que el modelo tiene un buen nivel de ajuste debido a que los indicadores cumplen con los criterios convencionales de fiabilidad y validez convergente ya que : 1) las relaciones de los indicadores con sus factores de carga hipotéticos son significativas $(p<0.001), 2)$ el modelo se ajusta al parámetro Bagozzi y Yi (1988), pues las cargas estandarizadas son mayores que el valor crítico de 0.50 y 3 . El promedio de las cargas estandarizadas de cada factor fue superior a 0.70 (Hair, Black, Babin y Anderson, 2010). Además, la consistencia interna de los constructos y los valores alfa de Cronbach exceden de acuerdo con Nunnally y Bernstein (1994) el valor mínimo aceptable de 0.70; asimismo los índices de fiabilidad compuesta (IFC) de cada factor son superiores al valor de referencia dado por Bagozzi y Yi (1988) de 0.60. Vale la pena resaltar además que el índice de varianza extraída (AVE) de cada factor supera el valor aceptable mínimo de 0.50 (Ab Hamid, Sami, y Sidek, 2017; Bagozzi y Yi, 1988; Fornell y Larcker, 1981). Por lo anterior se puede afirmar que el modelo presenta validez convergente. Para la validez discriminante se calcularon los indicadores: $\mathrm{BBNFI}=0.8722, \mathrm{BBNNFI}=$ $0.9121, \mathrm{CFI}=0.8765, \mathrm{IFI}=0.9145, \mathrm{MFI}=$ 0.912 y $\mathrm{RMSEA}=0.021$, lo que posibilita la confirmación de la fiabilidad, la validez convergente y la validez discriminante del modelo global de medida.

\section{Caracterización de la población de estudio}

En la Tabla 2 se presenta la estadística descriptiva de los 316 participantes de esta investigación, así como algunas características de las organizaciones en las que laboran. El $54 \%$ de las organizaciones que participaron son tipo mi Pymes. El 60\% de los participantes son hombres, un poco más del $55 \%$ cuenta con estudios superiores, aproximadamente el $75 \%$ son menores de 40 años y más del $80 \%$ lleva menos de 5 años en la organización.

Una vez establecida la fiabilidad y validez del modelo se realizó la contrastación de las hipótesis, cuyos 
Tabla 1.

Indicadores del Modelo de Medida

\begin{tabular}{|c|c|c|c|c|c|c|c|}
\hline \multirow[t]{2}{*}{ Factor } & \multirow[t]{2}{*}{ Ítem } & \multicolumn{3}{|c|}{ Fiabilidad } & \multicolumn{2}{|c|}{$\begin{array}{l}\text { Validez Convergente } \\
\text { Carga Factorial }\end{array}$} & \multirow{2}{*}{$\begin{array}{c}\text { Validez } \\
\text { Discriminante } \\
\text { Indicadores de } \\
\text { bondad } \\
\text { de ajuste del modelo } \\
\text { propuesto }\end{array}$} \\
\hline & & $\begin{array}{l}\text { Alfa de } \\
\text { Cronbach }\end{array}$ & IFC & AVE & $\square \square$ & $\square$ & \\
\hline \multirow{4}{*}{$\begin{array}{l}\text { Total, instrumento } \\
\text { relacióncon } \\
\text { clientes }\end{array}$} & & 0,9444 & & & Estandarizada & Promedio & \\
\hline & $\mathrm{rc} 1$ & $0,86775^{* *}$ & 0,843 & 0,5347 & 0,8154 & 0,777806667 & \\
\hline & $\mathrm{rc} 2$ & $0,74311^{* *}$ & 0,882 & & 0,71693 & & 0,95451 \\
\hline & $\mathrm{rc} 3$ & $0,76442^{* *}$ & 0,841 & & 0,80109 & & BBNNFI $\quad 0,942623$ \\
\hline Alfa del factor & & 0,95111 & 0,858 & & 0,81488 & & CFI $\quad 0,976809$ \\
\hline \multirow{3}{*}{$\begin{array}{l}\text { Relacióncon } \\
\text { proveedores }\end{array}$} & rp1 & $0,92605^{* *}$ & 0,866 & 0,56967 & 0,80384 & 0,77063667 & 0,953588 \\
\hline & $\mathrm{rp} 2$ & $0,72779^{* * *}$ & 0,853 & & 0,79726 & & 0,98814 \\
\hline & rp3 & $0,95796^{* *}$ & 0,858 & & 0,71081 & & RMSEA \\
\hline Alfa delFactor & & 0,90143 & 0,827 & & 0,79538 & & \\
\hline \multirow[t]{3}{*}{$\begin{array}{l}\text { Relacióncon } \\
\text { aliados }\end{array}$} & ral & $0,80389^{* * *}$ & 0,863 & 0,52214 & 0,78391 & 0,76049333 & \\
\hline & $\mathrm{ra} 2$ & $0,81164 * *$ & 0,884 & & 0,73511 & & \\
\hline & ra3 & $0,82465^{* *}$ & 0,815 & & 0,76246 & & \\
\hline Alfa del Factor & & 0,77975 & 0,826 & & 0,77443 & & \\
\hline \multirow[t]{3}{*}{ Reputación } & r1 & $0,90497 * *$ & 0,8623 & 0,51261 & 0,72479 & 0,77065 & \\
\hline & $\mathrm{r} 2$ & $0,72324^{* *}$ & 0,846 & & 0,7813 & & \\
\hline & r3 & $0,73247^{* *}$ & 0,847 & & 0,80586 & & \\
\hline Alfa del Factor & & 0,90052 & 0,821 & & 0,73257 & & \\
\hline \multirow[t]{2}{*}{ Red social } & rs1 & $0,73284 * *$ & 0,893 & 0,54067 & 0,76703 & 0,783385 & \\
\hline & rs2 & $0,95672^{* *}$ & 0,854 & & 0,79974 & & \\
\hline Alfa del Factor & & 0,95092 & 0,865 & & 0,73875 & & \\
\hline \multirow[t]{3}{*}{ Visión compartida } & $\mathrm{vc} 1$ & $0,84159^{* *}$ & 0,847 & 0,52745 & 0,8088 & 0,7902933 & \\
\hline & $\mathrm{vc} 2$ & $0,85925^{* *}$ & 0,825 & & 0,74564 & & \\
\hline & $\mathrm{vc} 3$ & $0,78295^{* *}$ & 0,892 & & 0,81644 & & \\
\hline Alfa del Factor & & 0,91229 & 0,855 & & 0,8023 & & \\
\hline \multirow[t]{2}{*}{ Confianza } & cf1 & $0,77669^{* *}$ & 0,827 & 0,61107 & 0,82703 & 0,774815 & \\
\hline & $\mathrm{cf} 2$ & $0,88445^{* *}$ & 0,878 & & 0,7226 & & \\
\hline Alfa del Factor & & 0,90779 & 0,835 & & 0,75664 & & \\
\hline \multirow[t]{6}{*}{ Red de valor } & rv1 & $0,83215^{* *}$ & 0,823 & 0,62602 & 0,78156 & 0,7808233 & \\
\hline & rv2 & $0,79515^{* *}$ & 0,822 & & 0,75362 & & \\
\hline & rv3 & $0,75512^{* *}$ & 0,870 & & 0,74235 & & \\
\hline & rv4 & $0,90655^{* *}$ & 0,880 & & 0,84137 & & \\
\hline & rv5 & $0,88179^{* *}$ & 0,827 & & 0,75573 & & \\
\hline & rv6 & $0,88449^{* *}$ & 0,872 & & 0,81031 & & \\
\hline Alfa del Factor & & 0,85596 & 0,863 & & 0,73052 & & \\
\hline
\end{tabular}

Fuente: $* \mathrm{p}<0.001 ; * *$ Cronbach's Alpha with missing item. Elaboración propia. 
Tabla 2.

Perfil de los sujetos participantes

\begin{tabular}{|c|c|c|c|c|c|c|c|c|c|c|c|}
\hline Sexo & $\%$ & Escolaridad & $\%$ & Edad & $\%$ & $\begin{array}{l}\text { Tipo de } \\
\text { contrato }\end{array}$ & $\%$ & $\begin{array}{c}\text { Antigüedad } \\
\text { en la } \\
\text { empresa }\end{array}$ & $\%$ & $\begin{array}{c}\text { Empresa } \\
\text { (\# empleados }\end{array}$ & $\%$ \\
\hline Hombre & $60 \%$ & Bachiller & $8,67 \%$ & de 18 a 25 & $6 \%$ & Término fijo & $13,4 \%$ & Menor 1 año & $36,48 \%$ & menos de 10 & $12 \%$ \\
\hline \multirow[t]{4}{*}{ Mujer } & $40 \%$ & Técnico & $11,7 \%$ & de 26 a 30 & $28.6 \%$ & Indefinido & $57.9 \%$ & Entre 1 y 3 años & $30,87 \%$ & & $12 \%$ \\
\hline & & Tecnólogo & $20,9 \%$ & de 31 a 40 & $39,4 \%$ & Outsourcing & $20.6 \%$ & De 3 a 5 años & $17,35 \%$ & 51 a 200 & $29 \%$ \\
\hline & & Universitario & $36,1 \%$ & de 45 a 60 & $21 \%$ & & & De 5 a 10 años & $9,95 \%$ & más de 200 & $46.2 \%$ \\
\hline & & Postgrado & $22,7 \%$ & más de 60 & $5 \%$ & & & Más de 10 años & $5,36 \%$ & & \\
\hline
\end{tabular}

Fuente: Elaboración propia.

resultados se presentan en la Tabla 3. Las siete hipótesis propuestas, así como las relaciones planteadas $\mathrm{H} 1, \mathrm{H} 2, \mathrm{H} 3$ y $\mathrm{H} 4$ son significativas con un nivel $\mathrm{p}<0.01$, y $\mathrm{H} 5$, $\mathrm{H} 6$ y H7 con un nivel $\mathrm{p}<0.001$, por lo que no son rechazadas. Asimismo, los indicadores de bondad de ajuste BBNFI, BBNNFI, CFI, IFI y MFI son mayores a 0.90 , y los residuales bajos (RMSEA= 0,047 ), de esta forma puede concluirse que la bondad del modelo de ajuste es adecuada. A través de los hallazgos se pudieron encontrar elementos que ponen de manifiesto el gran rol que se descarga sobre la creación de la red de valor en las empresas objeto de estudio. En términos generales los datos obtenidos señalan la importancia de cómo la buena gestión de las relaciones con clientes, proveedor y aliados, así como la construcción de ambientes de confianza pueden mejorar

Tabla 3.

Resultados del SEM para la Contrastación de Hipótesis

\begin{tabular}{|c|c|c|c|c|c|c|c|}
\hline \multicolumn{2}{|c|}{ Hipótesis } & \multicolumn{2}{|c|}{$\begin{array}{c}\text { Relación estructural } \\
\text { propuesta }\end{array}$} & \multicolumn{2}{|c|}{$\lambda($ estandarizada) } & \multirow{2}{*}{\multicolumn{2}{|c|}{$\begin{array}{c}\text { Resultado } \\
\text { No rechazada }\end{array}$}} \\
\hline \multicolumn{2}{|c|}{$\mathrm{H} 1$} & $\mathrm{RC} \rightarrow$ & Rep & & $58 * *$ & & \\
\hline \multicolumn{2}{|c|}{$\mathrm{H} 2$} & $\mathrm{RP} \rightarrow$ & Rep & & $23^{* *}$ & & No rechazada \\
\hline \multicolumn{2}{|c|}{ H3 } & $\mathrm{RA} \rightarrow$ & Rep & & $1 * * *$ & & No rechazada \\
\hline \multicolumn{2}{|c|}{$\mathrm{H} 4$} & $\mathrm{Vc} \rightarrow$ & Rs & & $9 * * *$ & & No rechazada \\
\hline \multicolumn{2}{|c|}{ H5 } & $\mathrm{Cf} \rightarrow$ & Rs & & $2 * * *$ & & No rechazada \\
\hline \multicolumn{2}{|c|}{ H6 } & Rep & RV & & $2 * * *$ & & No rechazada \\
\hline \multicolumn{2}{|c|}{$\mathrm{H} 7$} & $\mathrm{Rs} \rightarrow$ & $\mathrm{Rv}$ & & $3 * * *$ & & No rechazada \\
\hline \multirow{2}{*}{$\begin{array}{c}\text { Indicadores de } \\
\text { bondad de ajuste: }\end{array}$} & BBMFI & \multicolumn{2}{|c|}{ BBMFI } & CFI & IFI & MFI & RMSEA \\
\hline & 0,95451 & \multicolumn{2}{|c|}{0,942623} & 0,976809 & 0,9535 & 0,9881 & 0,047 \\
\hline
\end{tabular}

$* * \mathrm{p}<0.01, * * * \mathrm{p}<0.001$

Fuente: Elaboración propia. la reputación de la empresa y potenciar la construcción de su red social. Estos dos últimos, se convierten en activos intangibles, que tienen una poderosa influencia sobre clientes actuales y potenciales, por lo que su gestión no debe ser subestimada sobre todo en sectores de servicios relacionados con implementaciones tecnológicas. En este sentido la diversificación de canales de comunicación referidos a clientes, aliados y proveedores es primordial para proporcionar no sólo un aumento en el tiempo de relacionamiento, sino del intercambio económico, generando ingresos, empleo y recomendaciones de uso a otras personas y empresas, lo que sin duda impacta en la creación y resultados en la red de valor (Alama, López, Navas y Martin de Castro, 2009; Becerra,2008). 
La Figura 4 relaciona el modelo final contrastado con las cargas estructurales estandarizadas de las relaciones planteadas. De estos se puede concluir que tanto la Confianza (H5) $(0,422)$ como la visión compartida (H4) $(0,359)$ influyen directa, positiva y significativamente sobre la construcción del tejido o red social. Por su parte, las relaciones con el cliente $(\mathrm{H} 1)$ $(0,458)$, con proveedores $(\mathrm{H} 2)(0,423)$ y con aliados (H3) $(0,271)$, muestran una relación estadísticamente significativa con un nivel $\mathrm{p}<0.01$ con respecto a la variable Reputación. Las variables Reputación (H6) y Red Social (H7) presentan una relación significativa, directa y positiva de 0,312 y 0,213 respectivamente, frente a la competitividad a un nivel $\mathrm{p}<0.001$.

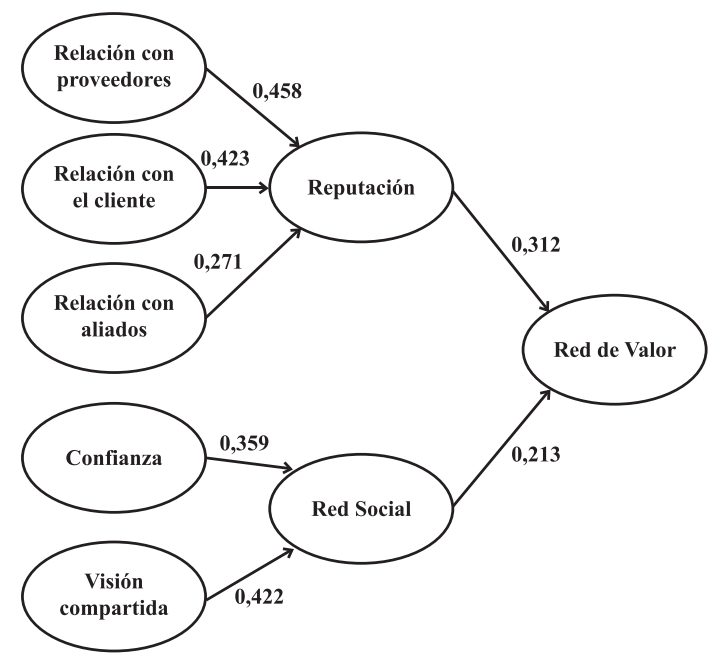

Figura 4. Modelo de Contrastación de hipótesis. Fuente: Elaboración propia.

\section{Conclusiones}

El capital relacional es importante mirarlo desde la cadena de valor, ya que la gestión de las organizaciones se sale de la compañía focal, entendida como aquella empresa que dinamiza la red mediante el ciclo logístico, el cual es atravesado por múltiples redes, aguas arriba o aguas abajo, dependiendo el lugar que cada uno ocupe en dicha red. En este sentido, al facultar a los empresarios en el trabajo organizado interno y externo, se pueden superar barreras que impiden el progreso, desarrollo y competitividad de pequeñas y medianas empresas en diversos sectores económicos, mediante estrategias que tienen que ver con el desarrollo de proveedores y relaciones de valor con clientes, que integren la innovación, la cultura organizacional, el trabajo en equipo y la gestión del conocimiento, tal como se identificó al analizar el instrumento (Garzón y Fisher, 2008).

A medida que se gestiona el capital relacional en las organizaciones y se empieza a documentar estas experiencias, se ve la relación que tiene con los otros capitales (Cousins, Handfield, Lawson y Petersen, 2006), avanzando en diversos factores que propician la innovación, representado en relaciones heterogéneas, dando paso a creaciones incomparables cargadas, de diseños, formas, colores, brindando soluciones que sobrepasan las necesidades y expectativas de los clientes en diversos mercados objetivos que tienen las organizaciones, los cuales están demandando bienes y servicios que brinden experiencias de valor, pero que estén en coherencia con las nuevas tendencias globales, como son: la sustentabilidad, el cuidado del medio ambiente, los productos eco sustentables, el manejo de energías limpias, el trabajo digno e inspirador, ya que no sólo los desarrollos tecnológicos que avanzan 
rápidamente cautivan o inclinan la opción de compra.

Como futuras líneas de investigación, se considera que vale la pena aplicar el estudio a sectores específicos para evaluar la gestión que al interior de los constructos se pueda dar en cada uno de ellos. También parece conveniente incluir una dimensión temporal en el modelo, debido a que el grado de relacionamiento con clientes proveedores y aliados, así como la construcción de ambientes de confianza y de compartimentación de la visión suelen ser a mediano o largo plazo. Por último, es conveniente que también se aborden la intención de compartir conocimiento tácito y explicito como una variable moderadora entre el relacionamiento y la reputación, a fin de establecer el impacto de las prácticas de gestión de conocimiento en la consolidación de la red de valor, asimismo sería necesario analizar las causas y los factores inhibidores de la adopción de prácticas de intercambio de conocimiento en la construcción de la red de valor.

\section{Referencias}

Ab Hamid, M., Sami, W., y Sidek, M. (2017). Discriminant validity assessment: Use of Fornell \& Larcker criterion versus HTMT criterion. Journal of Physics: Conference Series, 890(1), 121-163.

Ahumada, C. (2001). Teoría y cambio en las organizaciones. Un acercamiento desde los modelos de aprendizaje organizacional. Chile: Ediciones Universitaria de Valparaíso.

Aja, L. (2002). Gestión de información, gestión del conocimiento y gestión de la calidad en las organizaciones. Acimed, 10(5), 7- 8.
Alama, E., López, P., Navas, J., y Martin, G. (2009). El capital relacional como fuente de innovación tecnológica. Innovar, 19(35), 119-132.

Anderson, J. C., y Gerbing, D. W. (1988). Structural equation modeling in practice: A review and recommended two-step approach. Psychological bulletin, 103(3), 411.

Bagozzi, R. P., y Yi, Y. (1988). On the evaluation of structural equation models. Journal of the Academy of Marketing Science, 16, 74-94.

Becerra, F. (2008). Las redes empresariales y la dinámica de la empresa: aproximación teórica. Innovar, 18(32), 27-46

Blanchard, K. (2007). Liderazgo al más Alto Nivel. Bogotá, Colombia: Norma.

Brunet, L. (2009). El clima de trabajo en las organizaciones: definiciones, diagnóstico y consecuencias. México: Trillas.

Capello, R., y Faggian, A. (2005). Collective learning and relational capital in local innovation processes. Regional studies, 39(1), 75-87.

Ciprés, M. S., y Llusar, J. C. (2004). Concepto, tipos y dimensiones del conocimiento: configuración del conocimiento estratégico. Revista de economía y empresa, 22(52), 175-196.

Contreras, F., y Barbosa, D. (2013). Del liderazgo transaccional al liderazgo transformacional: implicaciones para el cambio organizacional. Revista Virtual Universidad Católica del Norte, 2(39), 152-164.

Cousins, P. D., Handfield, R. B., Lawson, B., y Petersen, K. J. (2006). Creating supply chain relational capital: the impact of formal and informal socialization processes. Journal of operations management, 24(6), 851-863. 
Dutton, J., y Duncan, R. (1987). The influence of the strategic planning process on strategic change. Strategic Management Journal, 8(2), 103-116.

Estrategia Magazine. (2018). La competitividad: La clave es la gente.

Figueroa, L. (2011). El aprendizaje organizacional desde una perspectiva evolutiva y constructivista de la organización. Revista de Psicología, 11(1), 139-148.

Fornell, C., y and Larcker, D., (1981). Evaluating Structural Equation Models with Unobservable Variables and Measurement Error. Journal of Marketing Research,18(1), $39-50$.

Gaitán, F. (2015). Estado-Universidad-Empresa: una relación que permite el desarrollo socio-científico en Colombia. Inclusión y Desarrollo, 3(1), 9-11.

Garzón. M, y Fisher. A, (2008) Modelo teórico de aprendizaje organizacional. Pensamiento y Gestión, (24),195-224.

Gattorna, J. (2009). Cadenas de abastecimiento dinámicas. Bogotá: Ecoe ediciones.

Gattorna, J. (2015). Dynamics Supply Chains: how to design, build and manage people-centric value networks. Boston: Prentice- Hall.

Gómez, D., Carranza, Y., y Ramos, C. (2016). Revisión documental, una herramienta para el mejoramiento de las competencias de lectura y escritura en estudiantes universitarios. Chakiñan, Revista de Ciencias Sociales y Humanidades, (1), 46-56.

Gómez, D., Carranza, Y., y Ramos, C. (2017a). Aportes de negociadores internacionales para el crecimiento, la innovación, el emprendimiento y la competitividad de las empresas colombianas. Revista Faccea,7(2),157-164.
Gómez, D. T., Carranza, Y., y Ramos, C. (2017b). Ecoturismo, turismo experiencial y cultural. Una reflexión con estudiantes de Administración de Turismo. Revista TEMAS, 3(11), 229 - 236.

Gómez, D., y Rincón, H. (2016). Perspectivas de cambio organizacional y aprendizaje en las organizaciones, una revisión de autores y teorías. Ponencia 51, Congreso Latinoamericano de Escuelas de Administración, CLADEA, ASCOLFA.

González, C. H., y Gálvez, A. É. (2008). Modelo de Emprendimiento en Red-MER. Aplicación de las teorías del emprendimiento a las redes empresariales. Revista Latinoamericana de Administración, (40), 13-31.

Hair, F., Black, C., Babin, BJ., y Anderson, E. (2010). Multivariate Data Analysis, Seventh Edition. New Jersey: Prentice Hall.

Kotler, P., y Armstrong, G. (2012). Marketing, Décimocuarta edición. México: Pearson Educación.

Lander, E. (2001). Los derechos de propiedad intelectual: en la geopolítica del saber de la sociedad global del conocimiento. Comentario Internacional: Revista del Centro Andino de Estudios Internacionales, (2), 79-88.

Likert, R. (1932). A technique for the measurement of attitudes. Archives of psychology.

Losada, J., Bonilla, M., y Gómez, D. (2018). Gestión del talento humano en las pequeñas empresas colombianas: una aproximación bibliográfica. CrearE. Cuadernos de Emprendimiento, (3), 62-69.

Mayorga, C., Ruiz, M., Mantilla, L., y Moyolema, M. (2015). Procesos de producción y productividad en la industria de calzado ecuatoriana: caso empresa Mabelyz. ECA Sinergia, 6(2), 88-100. 
Nonaka, I., y Takeuchi, H. (1999). La organización creadora de conocimiento: cómo las compañias japonesas crean la dinámica de la innovación. México: Oxford University Press.

Nunnally, J.C., y Bernstein, I.H. (1994). The Assessment of Reliability. Psychometric Theory, (3), 248-292.

Pérez, J., y Cortez J. (2009). Medición y validación del desempeño organizacional como resultado de acciones de aprendizaje. Revista Ciencias Estratégicas, 17(22), 251-271.

Polo, J. D., Fernández, M., Bargsted, M., Ferguson, L., y Rojas, M. (2017). The relationship between organizational commitment and life satisfaction: The mediation of employee engagement. Universia Business Review, (54), 110-145.

Rodríguez, M. I., y Alfaro, J. A. (2016). Innovación abierta en pymes proveedoras de automoción: una oportunidad para el desarrollo de nuevo producto. Universia Business Review, (50), 37-52.

Rubio, G. A., Téllez, C. A., y Gómez, D. T. (2019). Análisis de los factores que componen un sistema de gestión empresarial: estudio de caso para la dirección de vivienda. Revista Nacional De Administración, 10(2), 47-60. https://doi.org/10.22458/ rna.v10i2.2730

Spender, J. C. (1996). Making knowledge the basis of a dynamic theory of the firm. Strategic Management Journal, 17(S2), 45-62.

Teece, D. J. (1998). Capturing value from knowledge assets: The new economy, markets for know-how, and intangible assets. California Management Review, 40(3), 55-79.

Tejedo, F., y Ferraz. F. (2016). Información del Capital Humano: la generación de intangibles y la responsabilidad social. Cuadernos de Gestión, 16(1), 125-143.
Vargas, J., Villareal, F., López, P., y Rodríguez, P. (2014). Hacia un perfil del potencial de innovación en PYMES del Estado de Guanajuato. In Ciencias Administrativas y Sociales Handbook TV: Congreso Interdisciplinario de Cuerpos Académicos. ECORFAN, 10-23. 